\title{
Nanopatterned Titanium Implants Accelerate Bone Formation In Vivo
}

Andrew I.M. Greer, ${ }^{*}{ }^{\dagger}$ Vitali Goriainov, ${ }^{\dagger}$ Janos Kanczler, Cameron R.M. Black, Lesley-Anne Turner, Robert M.D. Meek, Karl Burgess, Ian MacLaren, Matthew J. Dalby, Richard O.C. Oreffo,* and Nikolaj Gadegaard*

Cite This: ACS Appl. Mater. Interfaces 2020, 12, 33541-33549

Read Online

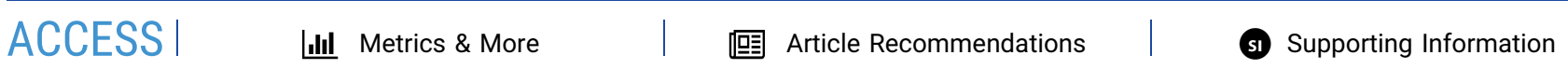

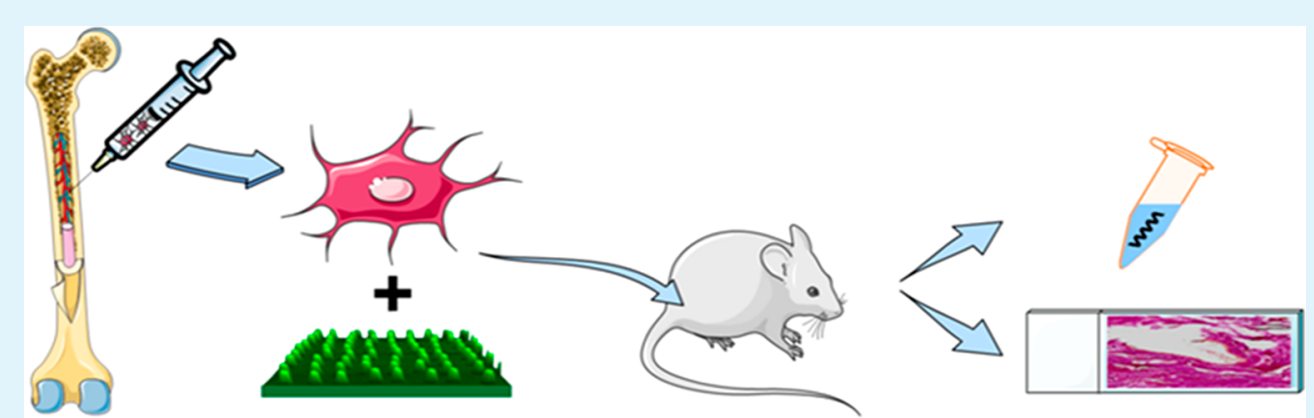

ABSTRACT: Accelerated de novo formation of bone is a highly desirable aim of implants targeting musculoskeletal injuries. To date, this has primarily been addressed by biologic factors. However, there is an unmet need for robust, highly reproducible yet economic alternative strategies that strongly induce an osteogenic cell response. Here, we present a surface engineering method of translating bioactive nanopatterns from polymeric in vitro studies to clinically relevant material for orthopedics: three-dimensional, large area metal. We use a titanium-based sol-gel whereby metal implants can be engineered to induce osteoinduction both in vitro and in vivo. We show that controlled disordered nanotopographies presented as pillars with $15-25 \mathrm{~nm}$ height and $100 \mathrm{~nm}$ diameter on titanium dioxide effectively induce osteogenesis when seeded with STRO-1-enriched human skeletal stem cells in vivo subcutaneous implantation in mice. After 28 days, samples were retrieved, which showed a 20 -fold increase in osteogenic gene induction of nanopatterned substrates, indicating that the sol-gel nanopatterning method offers a promising route for translation to future clinical orthopedic implants.

KEYWORDS: stem cell, osteogenesis, coating, prosthesis, sol-gel

\section{INTRODUCTION}

Arthroplasty is one of the greatest triumphs in modern surgery. In particular, the success of total hip replacement and its costeffectiveness in improving quality of life has led to it being named the "operation of the century". ${ }^{1-3}$ Furthermore, there are a number of factors placing an increasing demand on this procedure. The population is aging at an unprecedented pace, with the global proportion people aged 60 years or over predicted to grow to more than 2 billion by $2050 .{ }^{4}$ Concomitant with the aging population is the increased prevalence of age-related chronic disease (i.e., degenerative arthritis) and resultant functional impairment. The rate of revision surgery is expected to decrease with improved technology, yet as many as $26 \%$ of hip replacements fail within a decade of surgery. ${ }^{3}$ The increased age of the patient combined with inherent loss of bone occurring from the primary operation, or aberrant structural loading, severely reduces the success of any revision surgery., ${ }^{5-7}$
The load-bearing function of bone implants ensures the dominance of metal materials in the field, and titanium plays a critical role for various reasons. Titanium is biocompatible and lightweight but stronger than other alternative medical-implant metals such as steel or cobalt alloys. ${ }^{8}$ The excellent biocompatibility of titanium can be attributed to natural surface oxide (titanium dioxide or $\mathrm{TiO}_{2}$ ), where it provides an osteoconductive interface that attracts bone cells. ${ }^{9}$ Yet where cell recruitment is insufficient to address injury, there is a need for osteoinductive materials to stimulate. ${ }^{10}$

In recent years, nanotopographical modification of surfaces has been flagged by many research groups as a potentially

Received: June 5, 2020

Accepted: July 6, 2020

Published: July 6, 2020 

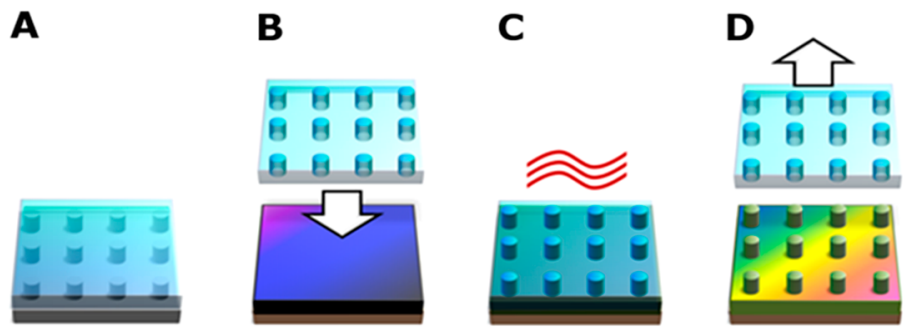

$\mathbf{E}$
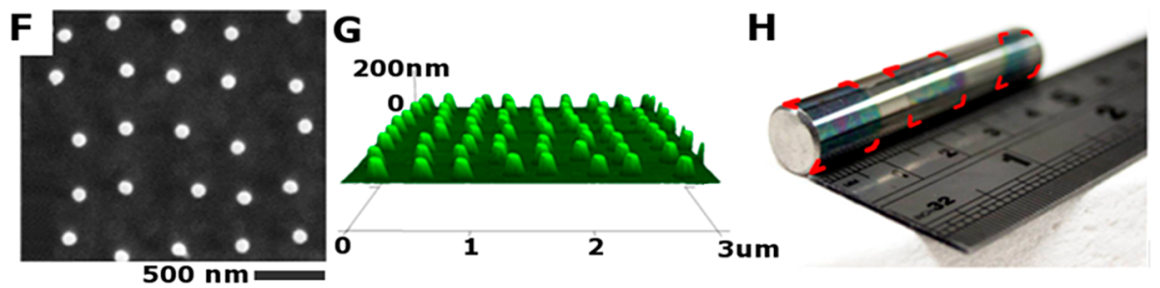

\section{I \\ $\mathbf{K}$}

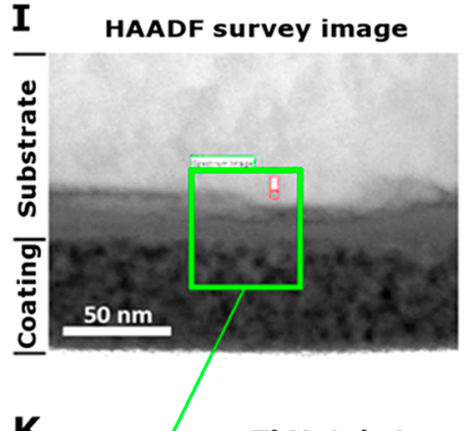

$\mathbf{J}$

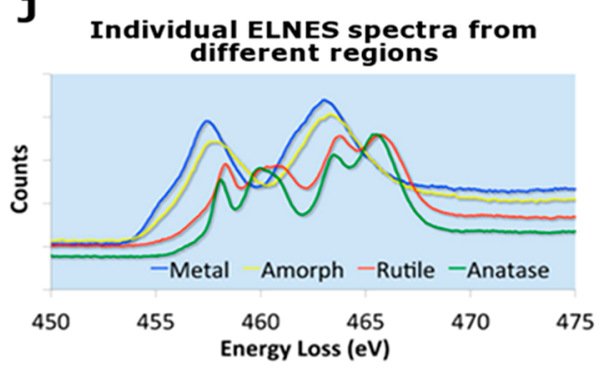

Ti Metal Amorphous Rutile-like Anatase Composite
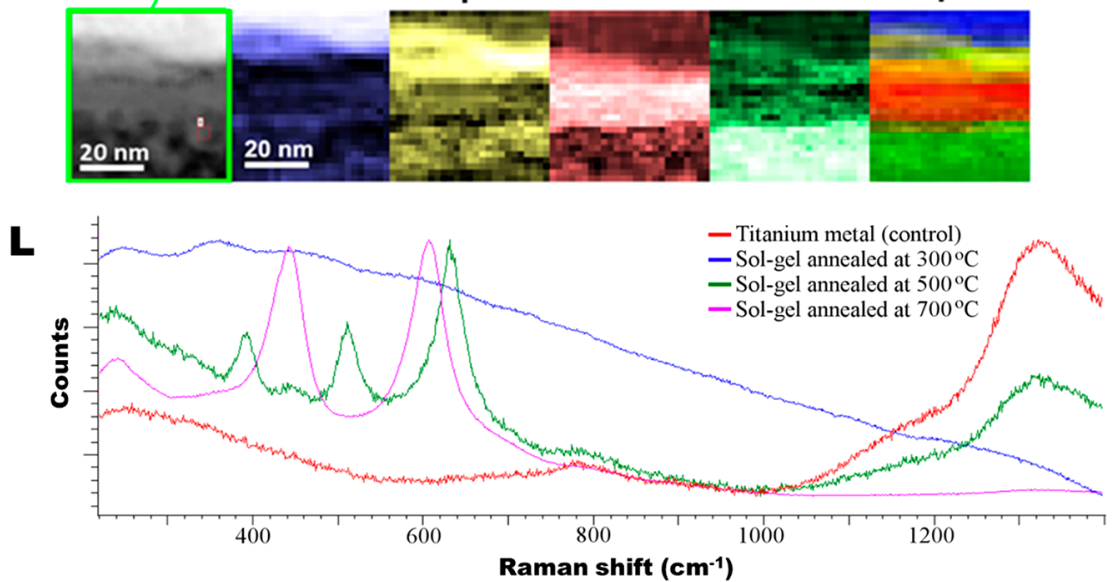

Figure 1. (A-E) Schematic diagram of nanopatterning $\mathrm{TiO}_{2}:$ (A) PDMS mold is cast from a silicon master, (B) sol-gel-coated substrate is imprinted with the PDMS stamp, (C) sandwich is cured before (D) releasing the stamp, (E) imprinted substrate is then annealed to form nanopatterned $\mathrm{TiO}_{2}$. (F-K) Surface characterization of formed $\mathrm{TiO}_{2}$. (F) Top-down SEM image of nanopillars, (G) Atomic force microscopybased 3D perspective of the nanopillars. $(\mathrm{H})$ Photograph of a $10 \mathrm{~mm}$ diameter cpTi rod with sol-gel coated surface featuring NSQ nanopillar arrays. The surface has undergone three iterations of stamping and each imprint is highlighted with superimposed red corners. (I) High-angle annular dark-field survey image of a cross-section from a sintered sol-gel-coated piece of cpTi. (J) Plot of internal energy-loss near-edge structure (ELNES) spectra from different regions of the subsection area. (K) Series of phase maps for the subsection site. For the single color images, the brighter a pixel, the stronger the fit to the appropriate spectrum displayed in the top right. The composite image is color mapped rather than brightness mapped, and the colors correspond to the ELNES key shown in part J. (L) Raman spectra from an accumulation of 10 traces per sample using a $785 \mathrm{~nm}$ wavelength laser in static mode on the Renishaw CCD sensor at 5 exposures per second.

effective modification of orthopedic implants. ${ }^{11-17}$ Cells sense surface texture via their adhesion receptor transmembrane, heterodimeric integrin. Integrin receptors have a footprint ca. $25 \mathrm{~nm}$ in diameter, with the final $5 \mathrm{~nm}$ binding to peptide ligands on the substrate (i.e., arginine, glycine, aspartic acid (RGD)). ${ }^{7}$ Disordered nanotextured substrates disrupt RGD ligand density and catalyze integrin clustering. The clustering induces macroscale focal adhesion formation. These macro- scale adhesions, composed of multiple nanoscale integrins, generate robust anchorage between the cell and the ECM, a necessity for osteogenesis. Thus, it is believed that this mechanism is responsible for the osteoinductive capacity of nanopatterned orthopedic devices. Such prosthetics bode reduced recovery time following implantation, extended lifetime of the device, and improved functionality. ${ }^{18,19}$ 
A

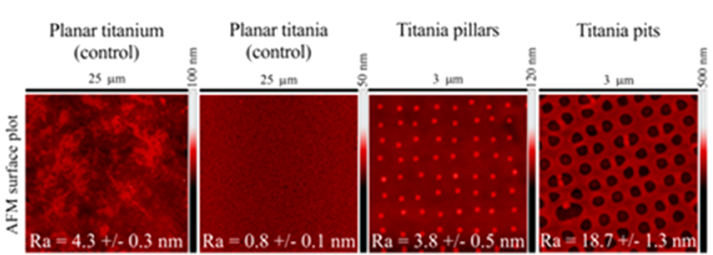

B

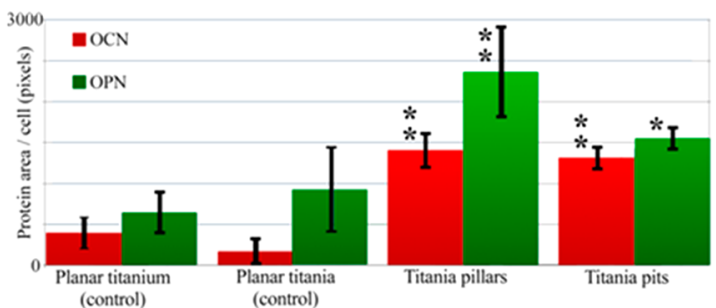

C

D
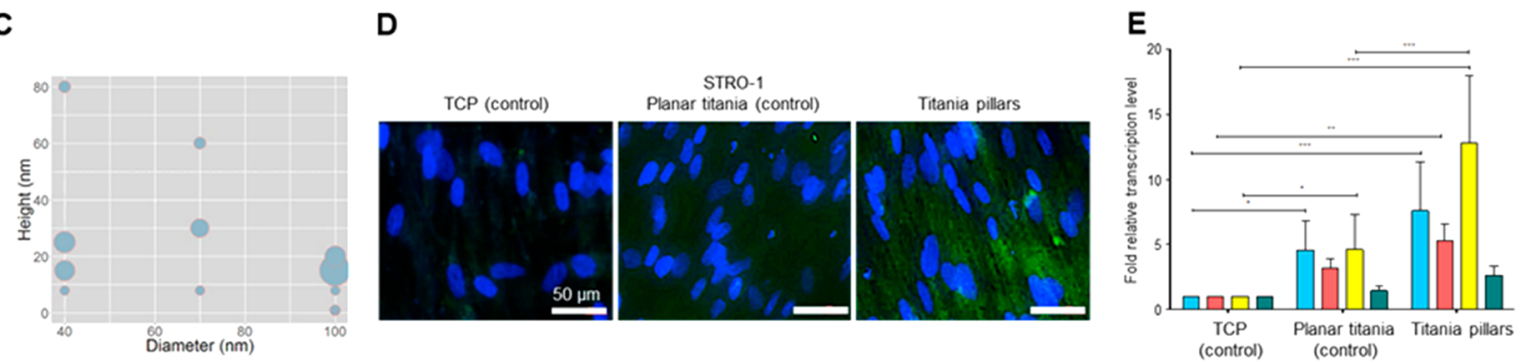

Figure 2. (A) AFM images of the tested surfaces with superimposed image Ra \pm standard deviation. The samples represent polished titanium, planar $\mathrm{TiO}_{2}$ surface derived from sintered sol-gel, NSQ nanopillars produced in sol-gel-derived $\mathrm{TiO}_{2}$ with $100 \mathrm{~nm}$ diameter, $15-25 \mathrm{~nm}$ tall pillars, and NSQ nanopits produced in sol-gel-derived $\mathrm{TiO}_{2}$ with $200 \mathrm{~nm}$ diameter, $60 \mathrm{~nm}$ deep pits. (B) OCN and OPN fluorescence levels from STRO-1 SSCs 21-day in vitro culture on the surfaces of panel A. (C) Bubble plot depicting the production of OCN for different pillar diameters and heights. (D) OPN immunofluorescence using a selective antihuman OPN antibody in STRO-1 SSCs after 21 days of in vitro culture. Note enhanced OPN protein expression on nanopillars in comparison to TCP and Titania planar substrates (blue-cell nuclei, green - OPN protein). (E) Real-time qPCR analysis of ALP (blue), collagen 1 (red), OPN (yellow), and OCN (green) in STRO-1 SSCs cultured in vitro on test surfaces and tissue culture plastic for 21 days. STRO-1 TCP is taken as a negative control. Results expressed as mean \pm SD, triplicate samples, individual experiment repeated five times, 2-way ANOVA test, $* p<0.05, * *_{p}<0.01, * * * p<0.001$.

There are few facile surface texturing strategies that possess even a moderate level of nanoscale control. One method is to anodize the surface, which under particular conditions can contain self-assembled nanopores. ${ }^{20}$ Park et al. ${ }^{20}$ and Brammer et $\mathrm{al}^{21}$ both concluded that sub-100 $\mathrm{nm}$ diameter pores are optimum for increased osteogenesis using anodization. However, with this approach, both depth and diameter are correlated, and the pore layout is not controlled. We have previously demonstrated that slightly disordered nanopits (referred to as NSQ) in polymeric substrates indeed are osteoinductive in vitro. $^{22}$ Sjostrom et al. have worked toward controlling the disorder of anodized nanofeatures via throughmask anodization. ${ }^{23}$ Using a block copolymer mask, the size of features may be controlled by the molecular weight of the polymer components and the spacing of micelles may be controlled through solvent evaporation. Despite this method exhibiting a high degree of control, the disorder scales directly with pitch during self-assembly, so although it is facile, it does not enable translation of nanopatterns with the electron-beam precision we previously demonstrated upon two-dimensional polymer substrates.

Aaritalo et $\mathrm{al}^{24}$ and Mendona et al. ${ }^{25}$ have shown that titania-based sol-gel processing has potential as a threedimensional coating process for metal-based orthopedic implants and both reiterated that nanoscale accuracy is important to trigger osteoblastic-specific gene expression. Eisenbarth et al. $^{26}$ found that $15 \mathrm{~nm} \mathrm{Ra}$ was the preferred roughness of annealed sol-gel coatings for adhesion strength and collagen I production. The aforementioned studies report that a sub- $8 \mathrm{~nm}$ difference in feature size will impact the osteogenesis. $^{8,20}$

We have previously demonstrated the ability to directly pattern titanium substrates by imprinting. ${ }^{27}$ However, the process was limited to small areas because of the forces required, and masters were fabricated in either diamond or sapphire. As the surface of any titanium implant is $\mathrm{TiO}_{2}$, we decided to take a different approach by producing our osteogenic nanotopography in a $\mathrm{TiO}_{2}$-based surface coating using a simple and scalable process. Through combining soft nanoimprint lithography with sol-gel processing, we have enabled a translational nanopatterning process capable of reproducing electron-beam-precise osteoinductive topographies upon nonplanar, large areas of clinically relevant, metal-based substrates.

\section{METHODS}

An NSQ patterned master was made in silicon using electron-beam lithography and reactive ion etching. ${ }^{22}$ The silicon master is not suitable to be used directly for pattern transfer, and thus a negative replica was made by casting polydimethylsiloxane (PDMS) against the silicon master (Figure 1A). PDMS has several important properties as a stamp material for this process. It has a naturally low surface energy, which aids the separation of the stamp from the substrate/implant after patterning, and it is flexible, further assisting this critical step. The next step in the process is to impart the NSQ pattern in the titanium-based sol-gel precursor, Figure 1B. As metal-based alkoxides cross-links in the presence of water, this reaction can be chemically stabilized and the viscosity of the solution be controlled by incorporating an organic solvent. Previous work by Yoon et al. ${ }^{28}$ and Richmond et al. ${ }^{29}$ used solvents with relatively high vapor pressures $\left(6-45 \mathrm{mmHg}\left(20^{\circ} \mathrm{C}\right)\right)$ leading to rapid evaporation of the solvent, reducing the handling time of the material during the patterning phase to a few seconds. To address this challenge, the solgel synthesized in this work featured titanium butoxide mixed with water, diethanolamine (stabilizing agent), and 1-hexanol. After spinning the titanium substrate with the sol-gel solution (Figure 1B), the PDMS stamp is imprinted into the gel and left to cure at 120 ${ }^{\circ} \mathrm{C}$ for $10 \mathrm{~min}$ (Figure 1C). After curing, the sol-gel is sufficiently mechanically stable to remove the stamp prior to sintering (Figure 1D, E). The combination of organic solvents in the sol-gel solution provide an extended handling time for reliable and reproducible patterning of the formed $\mathrm{TiO}_{2}$. Critically, the organic solvent mixture escapes the spin-cast film during the concomitant nanopatterning and 

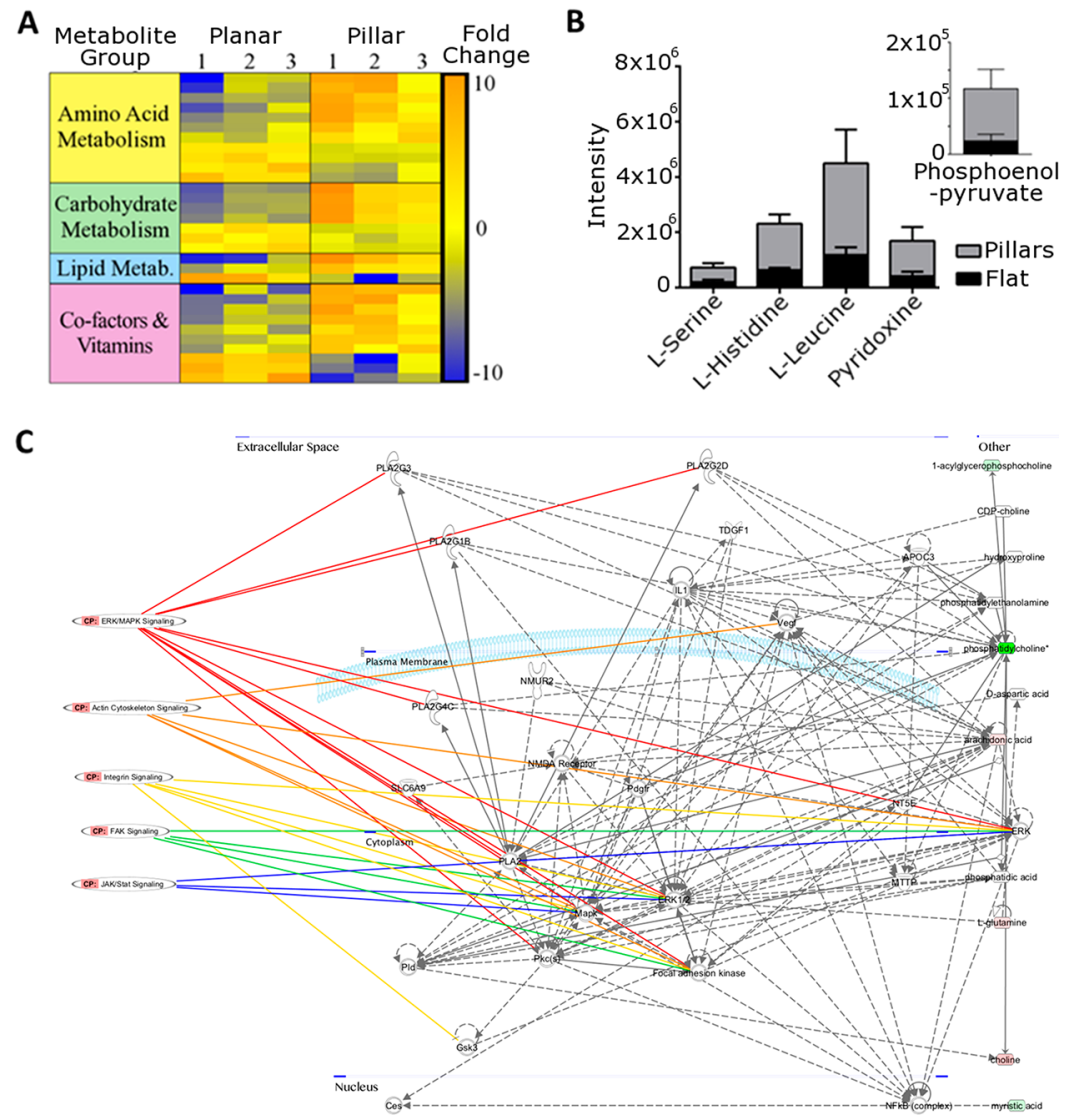

Figure 3. Metabolomic analysis reveals the higher metabolomic demand of SSC osteogenesis on nanopillar $\mathrm{TiO}_{2}$. (A) Heat map for a subset of identified metabolites highlighting difference in metabolite expression in cells cultured on nanopillars compared to planar TiO ${ }_{2}(\mathrm{increased}$ expression $=$ red, decreased expression $=$ blue $)$ characterized by group. (B) Stacked chart detailing intensities of the five most significantly $(t$ test with $P<0.05$ threshold) altered metabolites of those both matched to an authentic standard and showing a fold change of greater than 2 . Inset shows results for phosphoenolpyruvate for ease of viewing because of intensity scales. (C) Network map produced highlighting pathways involved in osteogenic and mechanotransductive signaling obtained from metabolomic analysis.

alkoxide reaction. This is facilitated by the permeability of PDMS to organic solvents and gases. ${ }^{30}$ Thus, formed $\mathrm{TiO}_{2}$ is left with nanosized pillars with high fidelity (Figure $1 \mathrm{~F}, \mathrm{G}$ ).

By capitalizing on the prolonged handling time of the novel sol-gel chemistry reported here, the imprinting process may accommodate multiple iterations of contact-print lithography upon nonplanar surfaces. Thus, a large area can be patterned through serial contactprinting. This is achieved by spin coating the sol-gel onto the stamp instead and exploiting the flexibility of the PDMS stamp to print nanopatterned sol-gel layers onto nonplanar, large areas of titanium. As an example, a $10 \mathrm{~mm}$ diameter commercially pure titanium (cpTi) rod was nanopatterned through three iterations of localized contactprinting (Figure $1 \mathrm{H}$ ).

A key requirement of the $\mathrm{TiO}_{2}$ film for any biomedical application is a firm integration with the underlying titanium substrate as to avoid any delamination. Scanning transmission electron microscopy (TEM) and energy-loss near-edge structure (ELNES) analysis were carried out on a cpTi sample containing the sol-gel coating which was sintered at $500{ }^{\circ} \mathrm{C}$. A high-angle annular dark-field (HAADF) survey image is displayed which provides a high-resolution image of the interface (Figure 1I). At the top of the cross-sectional image is the titanium substrate, and at the bottom the porous, sintered sol-gel layer. A subsection, as highlighted with the green box, was used for the phase analysis (Figure 1J). The ELNES spectrum shows a seamless integration between the annealed sol-gel and the underlying substrate (Figure 1K). Moreover, EELS also confirmed that the sol-gel layer had formed into a slightly porous almost entirely anatase phase, the preferential phase for orthopedic applications. ${ }^{31}$ Below the original cpTi surface, a dense layer of rutile-like crystals formed, with the interface to the metal containing a few nm of amorphous $\mathrm{TiO}_{2}$; this rutile/anatase layer of combined thickness about $40 \mathrm{~nm}$ possibly formed from oxidation of the metal during annealing.

\section{ANALYSIS AND DISCUSSION}

The chemical composition of the formed $\mathrm{TiO}_{2}$ surface using the sol-gel synthesis was examined using X-ray photoelectron spectroscopy (XPS, Table S1). Following sintering above 500 ${ }^{\circ} \mathrm{C}$, the carbon content $(5.5 \%)$ of formed $\mathrm{TiO}_{2}$ was observed to be comparable to the tested commercially pure titanium (cpTi) control (4.5\%). Using Raman spectroscopy, we found that the polymorph of the formed $\mathrm{TiO}_{2}$ could be controlled by the annealing temperature (Figure $1 \mathrm{~L}$ ). Annealing at $300{ }^{\circ} \mathrm{C}$ produced amorphous $\mathrm{TiO}_{2}$ and $500{ }^{\circ} \mathrm{C}$ produced anatase, whereas $700{ }^{\circ} \mathrm{C}$ formed rutile. On the basis of the preponderance in the literature and that both polymorphs have been reported to be biocompatible, the substrates manufactured in this study were annealed to form anatase. ${ }^{31}$ This not only ensures biocompatibility for a given biomedical device but also provides the ability to address specific crystal phases beneficial at the implant surface. ${ }^{30,32}$

During the annealing process, the sol-gel loses ca. $30 \%$ of its volume as a result of the organic material leaving the matrix. Consequently, this translates into dimensional changes of the nanopatterns from the imprint to the final sintered sample. We looked at the cell response across flat (control) substrates as compared to nanopatterned pits and pillars, Figure $2 \mathrm{~A}$. We 
A

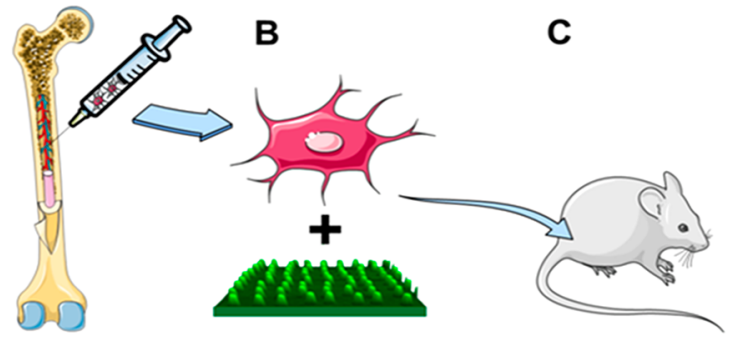

E

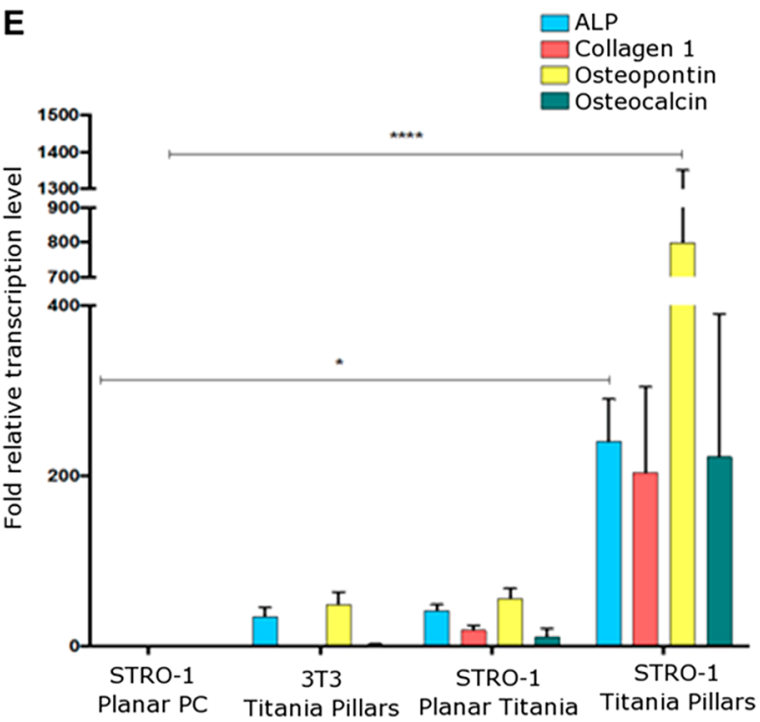

G
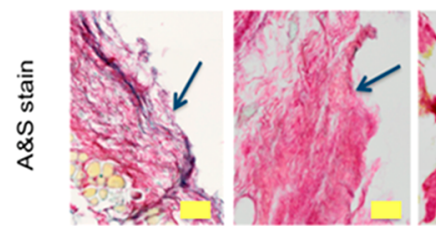

H

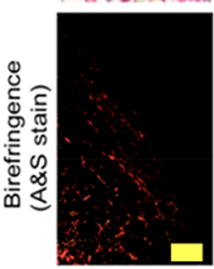

I

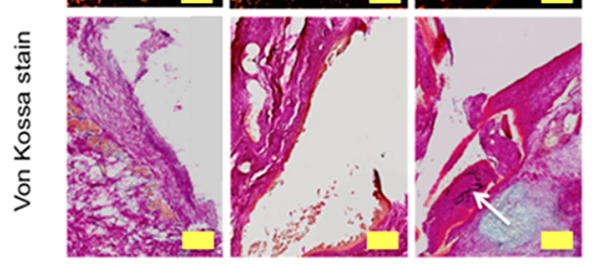

D
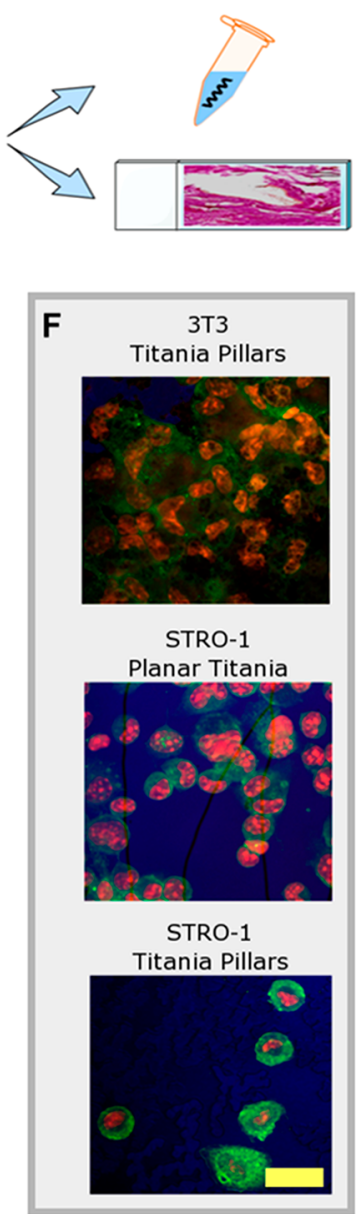

12 week chick femoral trabeculae

J

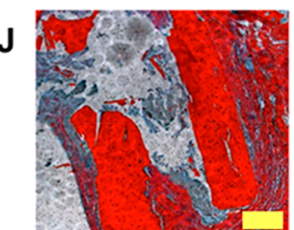

K

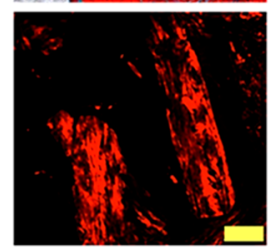

Figure 4. (A) Patient-derived SSCs were extracted from the bone marrow and (B) labeled with PKH26 prior to seeding on the substrates. (C) Cell-seeded implants were subcutaneously implanted in mice for 28 days. (D) Implant and the surrounding tissue were retrieved (E) Real-time qPCR analysis of bone marker genes ALP, collagen 1, OPN, and OCN from 4 separate mouse studies. STRO-1 SSCs cultured in vivo on test surfaces and controls for 28 days. Results are expressed as mean \pm SD, triplicate samples, individual experiments performed four times, two-way ANOVA test, $* * * p<0.001$. (F) OPN immunofluorescence of STRO-1 SSCs cultured in vivo for 28 days. Wide-spread polygonal morphology characteristic of osteoblasts can be observed in STRO-1 cells on $\mathrm{TiO}_{2}$ pillars (red fluorescence, nuclear counterstain; green, OPN protein). Scale bar $50 \mu \mathrm{m}$. (G) Alcian blue/Sirius red (A\&S) stain of the sections of tissue adjacent to substrate surfaces during in vivo incubation. The membrane that had been in contact with the surfaces (indicated with arrows) is attached to the subcutaneous adipose layer. There is an enhancement of Sirius red staining in the membrane that had been in contact with STRO-1 SSC-seeded nanopillar topography when compared to STRO-1 SSC-seeded planar surface or 3T3 SSC-seeded nanopillars. This enhancement of staining is indicative of collagen-rich matrix deposition. Scale bar $1 \mathrm{~mm}$. (H) Birefringence enhancement of A\&S-stained tissue sections. A significant augmentation of red-yellow birefringence in the membrane that had been in contact with STRO-1 SSC-seeded nanopillar topography is evident (arrow). Scale bar $1 \mathrm{~mm}$. (I) Von Kossa stain indicating that the areas of membrane shown to possess collagen-rich matrix are also developing foci of de novo mineralization, seen as black deposits of metallic silver (arrow). Scale bar $1 \mathrm{~mm}$. (J, K) A\&S stain and birefringence enhancement, respectively, of 12-week chick femoral bone trabeculae, provided for comparison. Scale bar $200 \mu \mathrm{m}$. 
found that control $\mathrm{TiO}_{2}$ substrates prepared from the sol-gel solution against a flat PDMS master, were significantly smoother than $\mathrm{CpTi}$. Here we found that bone markers (osteopontin (OPN) and osteocalcin (OCN)) from STRO-1 enriched human skeletal stem cells (SSCs) seeded on the materials were significantly higher on pillars as compared to other substrates, Figure 2B. With a change in polarity, as compared to previous studies, we also wanted to optimize the pillar dimensions to ensure the strongest response of the nanopattern. Hence a library of substrates with different dimensions (diameter and height) was manufactured in order to evaluate the optimal pattern geometry for skeletal stem cell differentiation on the $\mathrm{TiO}_{2}$ substrates. Here, pillars were fabricated to have a final diameter (after annealing) ranging from 40 to $100 \mathrm{~nm}$ and heights from 8 to $80 \mathrm{~nm}$. It is known that subtle changes in the height of nanopillars can change the cellular fate. ${ }^{33,34}$ The samples were seeded with SSCs and the expression of OCN relative to cell numbers was measured. It is notable that the pillars with a height of $15-30 \mathrm{~nm}$ exhibited the strongest responses, whereas the induction was less sensitive to the diameter, Figure $2 \mathrm{C}, \mathrm{D}$. The in vitro biological performance of nanopatterned $\mathrm{TiO}_{2}$ was corroborated by quantitative polymerase chain reaction (qPCR) analysis of bone specific markers alkaline phosphatase (ALP), collagen type I, OPN, and OCN, Figure 2E. Thus, nanopillars with a diameter of $100 \mathrm{~nm}$ and a height of $15-25 \mathrm{~nm}$ exhibited the highest levels of OPN and were thus chosen as the optimal design for the remainder of the study.

The reason $15-25 \mathrm{~nm}$ tall pillars are particularly osteogenic may be confirmed with further analysis. It is known that the ECM is a mesh of protein fibers including $10-300 \mathrm{~nm}$ diameter collagen and elastin. ${ }^{23}$ This nanoporous ECM mesh, which is continuously remodeled, is covered with adhesive proteins, notably fibronectin. ${ }^{24}$ Integrin receptors with a footprint of $23 \mathrm{~nm}$ transcend the cell membrane into the ECM and communicate surface texture information to the cell. It is proposed that the $15-25 \mathrm{~nm}$ tall, $100 \mathrm{~nm}$ diameter pillars are the optimum size for residing within the ECM mesh and anchoring integrin receptors. Disordered topographies are attributed to disrupting the RGD ligand distribution and inducing integrin clustering ${ }^{7 d}$. These integrin clusters are known to generate strong focal adhesions that supply the critical tension to support osteogenesis within cells.

We applied metabolomic analysis to further validate that the nanopillars functionally enhanced the osteogenic differentiation through expected pathways. Of the major metabolite subcategories, nucleotides, amino acids, carbohydrates, cofactors/vitamins increased the most in cells cultured on nanopillars with respect to planar substrates. Metabolic pathways were altered in cells cultured on nanopillars, with the greatest alterations observed in the aminoacyl tRNA biosynthesis pathway (Figure 3A). General upregulation in this pathway indicates increased protein production. Metabolism of amino acids such as L-histidine and L-serine was also drastically changed in nanopillars (Figure 3B). Both the increase in amino acid metabolism and protein synthesis support in vitro results, in which nanopillars promoted expression of OPN and OCN. These results mirror previous reports on increased metabolic demand by cells undergoing osteogenesis. ${ }^{15}$

Signaling pathways involved in mechanotransduction and bone differentiation/development were identified as critical cell responses to nanopillars (Figure 3C, Figures S4 and S5). Signaling components included: calcium, mitogen-activated protein kinases (MAPK) including extracellular signal-regulated kinases (ERK), ascorbic acid, and the JAK-STAT pathways, all of which are crucial in bone development. ${ }^{16}$ Identified pathways involved in extracellular substrate sensing and mechanotransduction such as integrins, focal adhesion kinase, and the actin cytoskeleton are known to influence osteogenic differentiation through focal adhesion dynamics. ${ }^{17}$ The metabolomic results provide further evidence that the nanotopographical cues on the $\mathrm{TiO}_{2}$ surface induce osseoinduction.

To provide preclinical evidence, we extracted human SSCs from bone marrow (Figure 4A) and seeded them on nanopillar $\mathrm{TiO}_{2}$ substrates (Figure 4B) prior to subcutaneous implantation in mice (Figure 4C). As a negative cellular control, fibroblasts were seeded on nanopillar substrates ahead of implantation. From the subcutaneous mouse model, we wanted to quantify the impact of the nanopillars by analyzing the change in bone-related gene markers and bone matrix protein synthesis and the early onset of bone tissue formation through histology. Thus, to identify the preseeded SSCs, we could track cells using the cell membrane label PKH26. This label was confirmed using FACS prior to seeding (Figure S6).

After 28 days in vivo, substrates were retrieved and FACS sorting of individual samples was undertaken to remove nonfluorescent contaminant cells of mouse origin (Figure S7). The purity of sorted isolated cell fractions was reanalyzed, and a relatively high degree of comparability was demonstrated between original and reanalyzed sorted cell fractions with retrieved cells retaining PKH26 fluorescence (Figure S8). Additional controls were carried out by analyzing gene expression of cell populations harvested from $\mathrm{TiO}_{2}$ nanopillars. Collected cells were either sorted or remained unsorted by FACS and analyzed by qPCR using specific human and mouse primers. A mixture of mouse and human cells (unsorted sample) demonstrated significantly higher expression of mouse compared to human osteogenic genes (Figure S9). Meanwhile, FACS sorted samples revealed enhanced expression of human osteogenic genes in excess to the host, thus validating the relative purity of the FACS sorted sample. Across four in vivo studies, there was significant enhancement of bone-related gene expression in SSCs cultured on $\mathrm{TiO}_{2}$ nanopillars compared to $\mathrm{PC}$ planar and planar $\mathrm{TiO}_{2}$ controls, or to fibroblasts on $\mathrm{TiO}_{2}$ nanopillars (Figure 4E). Retrieved and sorted in vivo cell populations were quantitatively analyzed for ALP, collagen 1, OPN, and OCN expression pooled from four separate mouse studies (Figure S10).

Cells displaying a spread polygonal morphology (characteristic of osteoblasts expressing OPN) were observed on $\mathrm{TiO}_{2}$ pillars (Figure 4F). The intensity of OPN fluorescence in SSCs cultured on nanopillars was significantly enhanced compared to cells on planar surfaces and fibroblasts on nanopillars. To determine if osteogenic gene activation and bone matrix protein expression in vivo was linked with histological evidence of de novo osteogenesis triggered by substrate surface topographies, the tissue membrane developed subcutaneously by mouse hosts to envelop cell-seeded substrates during in vivo incubation was examined. When compared to controls, Sirius red/Alcian blue staining of the membrane in contact with $\mathrm{TiO}_{2}$ nanopillars demonstrated augmented deposition of collagen in extracellular matrix (Figure 4G, H). Further birefringence enhancement of the areas containing the mineralization foci indicated collagen fibrils coaligned into bundles showing strong red-yellow birefringence (Figure $4 \mathrm{H}$ ), which had been 
suggested to be characteristic of type I collagen, ${ }^{35}$ whereas the orientation of discrete bundles was heterogenic and organized in different planes. The Sirius red/Alcian blue stain of SSC on nanopillars were comparable with that of developing bone from 12-week-old chick embryo (Figure 4J, K). Meanwhile, von Kossa stain indicated initial foci of mineralization observed only on nanopillar substrates (Figure 4I).

\section{CONCLUSIONS}

We have shown that the developed sol-gel (which exhibits a potential processing window $900 \%$ longer than similar chemistries reported previously ${ }^{28}$ ) is effective in transferring features in a nonstringent, high throughput manner onto the surface of titanium. Through the synthesis of sol-gel coupled with the versatile $3 \mathrm{D}$ nanopattering process, thin films of $\mathrm{TiO}_{2}$ have been realized with the capacity to incorporate highly precise nanofeatures of either positive or negative tone. Previously the production of nanopillars via direct embossing was considered a stringent process and as a consequence biological analysis of pillar topographies had proved challenging. $^{16,27}$ The flexibility of the PDMS stamp allows the patterning to be applied to nonplanar surfaces with good compliance, to the extent that bulk metal rods have been patterned around their outer circumference. The in vitro and in vivo studies indicate that $15-25 \mathrm{~nm}$ tall nanopillars with a diameter of $100 \mathrm{~nm}$ and in a disordered geometric layout provide an enhanced osteoinductive platform in comparison to planar or pitted surfaces. OPN is known to be produced by cells late in the bone formation process ${ }^{36}$ and analysis of OPN levels between nanopits and nanopillars indicated that the nanopillars expressed approximately 50\% more per cell than nanopits. Metabolomic pathway analysis indicated that osteogenic biochemical pathways are exclusively activated on the pillar surfaces, as are mechanotransduction pathways involving integrin signaling. Human SSC in vivo cultures on nanopillar substrates in subcutaneous mouse model revealed a 20 -fold increase in bone marker gene activation as compared to flat control, and this process was linked to bone matrix protein synthesis, evidence of enhanced bone tissue formation and early mineralization. The results described here suggest exciting opportunities for the use of fine-tuned surface engineering in the investigations of the effects the nanopatterned surfaces exert on skeletal cell fate, phenotype, and function, as well as potential translation to clinical application in implantology targeting enhanced osseointegration.

\section{EXPERIMENTAL SECTION}

Sol-Gel Synthesis. All chemicals were sourced from SigmaAldrich. The sol-gel solution was prepared by mixing $0.96 \mathrm{~mL}$ of diethanolamine (99\%) with $5.54 \mathrm{~mL}$ of 1-hexanol (99\%) and $0.10 \mathrm{~mL}$ of deionized water. The mixture was vigorously stirred for $10 \mathrm{~min}$ and adding $3.40 \mathrm{~mL}$ of $\mathrm{Ti}(\mathrm{OBu})_{4}$ (97\%) while stirring.

Stamp Fabrication. Electron beam lithography (EBL) and reactive ion etching were deployed to produce a mold for PDMS casting. Sylgard 184 (Dow Corning) was cast upon the molds in a 1:10 curing agent:monomer weight ratio, cured at $70{ }^{\circ} \mathrm{C}$ overnight, before being peeled off.

Imprinting. Titanium samples were spin-coated with sol-gel at $9000 \mathrm{rpm}$ for $7 \mathrm{~s}$. PDMS stamps were then placed on to the sol-gel coating at a pressure of $44 \mathrm{~Pa}$ and baked with the stamp present at $120^{\circ} \mathrm{C}$ for $10 \mathrm{~min}$. The PDMS stamp was removed, and the titanium samples were sintered at $500{ }^{\circ} \mathrm{C}$ with a ramp rate of $2{ }^{\circ} \mathrm{C} / \mathrm{min}$ to obtain $\mathrm{TiO}_{2}$.
Cell Culture. Human CD271+ (magnetic isolation kit from Stem Cell Technologies, UK) osteoprogenitor cells were enriched from bone marrow samples. Cells were cultured using Dulbecco's modified Eagle's medium supplemented with fetal bovine serum, penicillin streptomycin, nonessential amino acids, sodium pyruvate, and Lglutamine (all sourced from Sigma-Aldrich). The cells were cultured upon the samples for 3 weeks, at the end of which the samples were fluorescently tagged to identify the cell nucleus (DAPI stain), cell cytoskeleton (actin stain), and bone-related proteins OCN and OPN. Details on fluorescence setup may be found in the supplementary pages.

qPCR Analysis. Cells were released from relevant culture surfaces (eight material replicates for in vitro and six replicates for in vivo experiments) using Trypsin-EDTA buffer, Sigma-Aldrich, and lysed. Total mRNA extraction was performed using the Qiagen RNeasy kit according to manufacturer's instructions. mRNA samples were treated with DNase and reverse-transcribed using SuperScript first-strand synthesis system (Veriti Thermal Cycler, Applied Biosystems). Realtime qPCR using SYBR Select Master Mix (Life Technologies) was accomplished on a 7500 Real-Time PCR system (Applied Biosystems) for expression of $\beta$-actin, ALP, collagen 1, OPN, and OCN genes. Further details may be found in the Supporting Information.

Metabolomic Analysis. Nanopillar $\mathrm{TiO}_{2}$ and planar $\mathrm{TiO}_{2}$ controls were used for metabolomic analysis. Cells were cultured for 14 days on different substrates. On day 14, samples were washed in phosphate buffered saline and metabolites extracted by shaking samples in a chloroform/methanol/water solution for $1 \mathrm{~h}$ before centrifuging at $13000 \mathrm{~g}$ for $5 \mathrm{~min}$ at $4{ }^{\circ} \mathrm{C}$ in order to remove cell debris. Metabolites were analyzed using hydrophilic interaction liquid chromatography-mass spectrometry (ZIC-pHILIC (Merck Sequant) and Orbitrap Exactive (Thermo Fisher Scientific)) with a $15 \mathrm{~min}$ gradient running from $80 \%$ acetonitrile $/ 20 \% \mathrm{H}_{2} \mathrm{O}$ to $20 \%$ acetonitrile/80\% $\mathrm{H}_{2} \mathrm{O}$ and a mass range of between 70 and 1400 $\mathrm{Da}$ in positive/negative ionization switching mode. Metabolites were identified against mass and retention times of known standards or predicted retention time using the authentic standards as a seed using the $\mathrm{IDEOM}^{24} / \mathrm{MzMatch}^{25}$ pipeline. Metabolite data were processed using IDEOM, Metaboanalyst $2.0^{26}$ and IPA, Qiagen (for IPA all identified metabolites were uploaded). Metabolites matched to authentic standards.

In Vivo Cell Culture. Only passage 1 cells were used. SSCs were seeded at $220 / \mathrm{cm}^{2}$ density labeled with PKH26 (Sigma-Aldrich), the staining reaction stopped with FCS, and the cells pelleted and washed in $\alpha$-MEM. The cells were analyzed by flow cytometry, seeded onto appropriate substrates, and cultured in vitro for 2 days to ensure cell adherence to the substrates. The substrates were imaged prior to implantation subcutaneously into male nude mice bilaterally. After 4 weeks, implants were retrieved, substrates dissected from enveloping host tissue and further imaging undertaken. Substrates were rinsed in PBS and cells released using Trypsin-EDTA buffer (Sigma-Aldrich), resuspended in PBS and sorted on a FACSAria II cell sorter (BD Biosciences) to separate original PKH26 stained fluorescent human SSC fraction from mouse (host) cells. Cells were pelleted, lysed, and examined for $\mathrm{qPCR}$ of bone marker gene expression as described above.

Ethics. All animal experimentation was performed and approved under license from the Home Office in accordance with the Animals (Scientific Procedures) Act (1986). All mice were raised within the University of Southampton Biomedical Research Facility and were housed in appropriate environments in rooms maintained at $22 \pm 2$ ${ }^{\circ} \mathrm{C}$ with a $12 \mathrm{~h}$ light: $12 \mathrm{~h}$ dark cycle (PPL 30/2880), (LREC194/99/ $1)$.

\section{ASSOCIATED CONTENT}

\section{Supporting Information}

The Supporting Information is available free of charge at https://pubs.acs.org/doi/10.1021/acsami.0c10273. 
Additional figures for fluorescence analysis, material quantification, nanopattern optimization, qPCR, flow cytometry, metabolomics, and additional experimental setup details (PDF)

\section{AUTHOR INFORMATION}

\section{Corresponding Authors}

Andrew I.M. Greer - Division of Biomedical Engineering, School of Engineering, University of Glasgow, Glasgow G12 8LT, United Kingdom; 이이.org/0000-0003-2817-5884; Email: Andrew.Greer@glasgow.ac.uk

Nikolaj Gadegaard - Division of Biomedical Engineering, School of Engineering, University of Glasgow, Glasgow G12 8LT, United Kingdom; 이이.org/0000-0002-3396-846X; Email: Nikolaj.Gadegaard@glasgow.ac.uk

Richard O.C. Oreffo - Bone and Joint Research Group, Centre for Human Development Stem Cells and Regeneration, University of Southampton, Southampton SO16 6YD, United Kingdom; Email: Richard.Oreffo@soton.ac.uk

\section{Authors}

Vitali Goriainov - Bone and Joint Research Group, Centre for Human Development Stem Cells and Regeneration, University of Southampton, Southampton SO16 6YD, United Kingdom

Janos Kanczler - Bone and Joint Research Group, Centre for Human Development Stem Cells and Regeneration, University of Southampton, Southampton SO16 6YD, United Kingdom

Cameron R.M. Black - Bone and Joint Research Group, Centre for Human Development Stem Cells and Regeneration, University of Southampton, Southampton SO16 6YD, United Kingdom

Lesley-Anne Turner - Centre for Cell Engineering, University of Glasgow, Glasgow G12 8QQ United Kingdom

Robert M.D. Meek - Department of Orthopaedics, Queen Elizabeth University Hospital, Glasgow, Lanarkshire G51 4TF, United Kingdom

Karl Burgess - Glasgow Polyomics Facility, Institute of Biomedical and Life Sciences, University of Glasgow, Glasgow G12 8QQ, United Kingdom

Ian MacLaren - School of Physics, University of Glasgow, Glasgow G12 8QQ, United Kingdom; 이이.org/00000002-5334-3010

Matthew J. Dalby - Centre for Cell Engineering, University of Glasgow, Glasgow G12 8QQ United Kingdom; (1) orcid.org/ 0000-0002-0528-3359

Complete contact information is available at:

https://pubs.acs.org/10.1021/acsami.0c10273

\section{Author Contributions}

${ }^{\dagger}$ A.I.M.G. and V.G. contributed equally to this work.

\section{Funding}

N.G., M.J.D., and R.O.C.O. acknowledge the support of the MRC through Grant G1000842 and BBSRC through Grants BB/K011235/1 and BB/L021072/1. A.I.M.G. was funded by the EPSRC DTA student allocation.

\section{Notes}

The authors declare no competing financial interest.

\section{ACKNOWLEDGMENTS}

We are grateful for the support and training provided by the JWNC technical staff for all the fabrication work. Dr. Rasmus
Pedersen and Carol-Anne Smith are acknowledged for assistance with the preparation of samples.

\section{REFERENCES}

(1) Chang, R. W.; Pellissier, J. M.; Hazen, G. B. A cost-effectiveness analysis of total hip arthroplasty for osteoarthritis of the hip. JAMA 1996, 275 (11), 858-865.

(2) Wright, G. M.; Porteous, M. J. An inconvenient truth. Bone Joint 360 2015, 4 (1), 2-5.

(3) Learmonth, I. D.; Young, C.; Rorabeck, C. The operation of the century: total hip replacement. Lancet 2007, 370 (9597), 1508-1519.

(4) Lakin, K. C.; Burke, M. M. Looking forward: Research to respond to a rapidly aging population. Res. Pract Persons Severe Disabl 2019, 44 (4), 280-292.

(5) Houdek, M. T.; Witten, B. G.; Hevesi, M.; Griffin, A. M.; Salduz, A.; Wenger, D. E.; Sim, F. H.; Ferguson, P. C.; Rose, P. S.; Wunder, J. $\mathrm{S}$. Advancing patient age is associated with worse outcomes in lowand intermediate-grade primary chondrosarcoma of the pelvis. J. Surg. Oncol. 2020, 121 (4), 638-644.

(6) Xu, K.; He, R.; Zhang, Y.; Qin, S.; Wang, G.; Wei, Q.; Zhang, H.; Ji, F. Forsythiaside inhibited titanium particle-induced inflammation via the NF- $\kappa \mathrm{B}$ signaling pathway and RANKL-induced osteoclastogenesis and titanium particle-induced periprosthetic osteolysis via JNK, p38, and ERK signaling pathways. RSC Adv. 2019, 9 (22), 12384-12393.

(7) Goodman, S. B.; Gallo, J. Periprosthetic Osteolysis: Mechanisms, Prevention and Treatment. J. Clin. Med. 2019, 8 (12), 2091.

(8) Sjöström, T.; McNamara, L. E.; Meek, R.; Dalby, M. J.; Su, B. 2D and 3D Nanopatterning of Titanium for Enhancing Osteoinduction of Stem Cells at Implant Surfaces. Adv. Healthcare Mater. 2013, 2 (9), 1285-1293.

(9) Cui, C.; Liu, H.; Li, Y.; Sun, J.; Wang, R.; Liu, S.; Lindsay Greer, A. Fabrication and biocompatibility of nano- $\mathrm{TiO} 2 /$ titanium alloys biomaterials. Mater. Lett. 2005, 59 (24-25), 3144-3148.

(10) Lee, H. W.; Kim, S. Y.; Kim, A. Y.; Lee, E. J.; Choi, J. Y.; Kim, J. $B$. Adiponectin stimulates osteoblast differentiation through induction of COX2 in mesenchymal progenitor cells. Stem Cells 2009, 27 (9), 2254-2262.

(11) Biggs, M. J.; Richards, R. G.; Gadegaard, N.; Wilkinson, C. D.; Oreffo, R. O.; Dalby, M. J. The use of nanoscale topography to modulate the dynamics of adhesion formation in primary osteoblasts and ERK/MAPK signalling in STRO-1+ enriched skeletal stem cells. Biomaterials 2009, 30 (28), 5094-5103.

(12) Biggs, M. J. P.; Richards, R. G.; Dalby, M. J. Nanotopographical modification: a regulator of cellular function through focal adhesions. Nanomedicine 2010, 6 (5), 619-633.

(13) Chen, Z.; Bachhuka, A.; Wei, F.; Wang, X.; Liu, G.; Vasilev, K.; Xiao, Y. Nanotopography-based strategy for the precise manipulation of osteoimmunomodulation in bone regeneration. Nanoscale 2017, 9 (46), 18129-18152.

(14) Dalby, M. J.; Gadegaard, N.; Oreffo, R. O. Harnessing nanotopography and integrin-matrix interactions to influence stem cell fate. Nat. Mater. 2014, 13 (6), 558-569.

(15) Dalby, M. J.; Gadegaard, N.; Tare, R.; Andar, A.; Riehle, M. O.; Herzyk, P.; Wilkinson, C. D.; Oreffo, R. O. The control of human mesenchymal cell differentiation using nanoscale symmetry and disorder. Nat. Mater. 2007, 6 (12), 997-1003.

(16) Gadegaard, N.; Dalby, M. J.; Riehle, M. O.; Wilkinson, C. D. Optimizing substrate disorder for bone tissue engineering of mesenchymal stem cells. J. Vac Sci. Technol. B: Microelectron Nanometer Struct 2008, 26 (6), 2554-2557.

(17) McNamara, L. E.; Sjostrom, T.; Burgess, K. E.V.; Kim, J. J.W.; Liu, E.; Gordonov, S.; Moghe, P. V.; Meek, R.M. D.; Oreffo, R. O.C.; $\mathrm{Su}$, B.; Dalby, M. J. Skeletal stem cell physiology on functionally distinct titania nanotopographies. Biomaterials 2011, 32 (30), 74037410.

(18) Frandsen, C. J.; Noh, K.; Brammer, K. S.; Johnston, G.; Jin, S. Hybrid micro/nano-topography of a $\mathrm{TiO} 2$ nanotube-coated commer- 
cial zirconia femoral knee implant promotes bone cell adhesion in vitro. Mater. Sci. Eng., C 2013, 33 (5), 2752-2756.

(19) Klymov, A.; Prodanov, L.; Lamers, E.; Jansen, J. A.; Walboomers, X. F. Understanding the role of nano-topography on the surface of a bone-implant. Biomater. Sci. 2013, 1 (2), 135-151.

(20) Park, J.; Bauer, S.; von der Mark, K.; Schmuki, P. Nanosize and vitality: $\mathrm{TiO} 2$ nanotube diameter directs cell fate. Nano Lett. 2007, 7 (6), 1686-1691.

(21) Brammer, K. S.; Oh, S.; Cobb, C. J.; Bjursten, L. M.; Heyde, H. v. d.; Jin, S. Improved bone-forming functionality on diametercontrolled TiO2 nanotube surface. Acta Biomater. 2009, 5 (8), 32153223.

(22) Dalby, M. J.; Gadegaard, N.; Tare, R.; Andar, A.; Riehle, M. O.; Herzyk, P.; Wilkinson, C. D. W.; Oreffo, R. O. C. The control of human mesenchymal cell differentiation using nanoscale symmetry and disorder. Nat. Mater. 2007, 6 (12), 997-1003.

(23) Sjöström, T.; McNamara, L. E.; Yang, L.; Dalby, M. J.; Su, B. Novel anodization technique using a block copolymer template for nanopatterning of titanium implant surfaces. ACS Appl. Mater. Interfaces 2012, 4 (11), 6354-6361.

(24) Ääritalo, V.; Areva, S.; Jokinen, M.; Lindén, M.; Peltola, T. Solgel-derived $\mathrm{TiO} 2-\mathrm{SiO} 2$ implant coatings for direct tissue attachment. Part I: design, preparation and characterization. J. Mater. Sci.: Mater. Med. 2007, 18 (9), 1863-1873.

(25) Mendonca, G.; Mendonca, D. B.S.; Simoes, L. G. P.; Araujo, A. L.; Leite, E. R.; Duarte, W. R.; Aragao, F. J.L.; Cooper, L. F. The effects of implant surface nanoscale features on osteoblast-specific gene expression. Biomaterials 2009, 30 (25), 4053-4062.

(26) Eisenbarth, E.; Velten, D.; Breme, J. Biomimetic implant coatings. Biomol. Eng. 2007, 24 (1), 27-32.

(27) Greer, A.; Seunarine, K.; Khokhar, A.; Li, X.; Moran, D.; Gadegaard, N. Direct nanopatterning of commercially pure titanium with ultra-nanocrystalline diamond stamps. Phys. Status Solidi A 2012, 209, 1721-1725.

(28) Yoon, K.-m.; Yang, K.-y.; Lee, H. Fabrication of polycrystalline $\mathrm{TiO} 2$ nanopatterns by $\mathrm{TiO} 2$ sol base imprint lithography. Thin Solid Films 2009, 518 (1), 126-129.

(29) Richmond, D. A.; Zhang, Q.; Cao, G.; Weiss, D. N. Pressureless nanoimprinting of anatase $\mathrm{TiO} 2$ precursor films. J. Vac. Sci. Technol., B: Nanotechnol. Microelectron.: Mater., Process., Meas., Phenom. 2011, 29 (2), 021603-021603-5.

(30) Abate, A. R.; Lee, D.; Do, T.; Holtze, C.; Weitz, D. A. Glass coating for PDMS microfluidic channels by sol-gel methods. Lab Chip 2008, 8 (4), 516-518.

(31) Chen, D.; Jordan, E. H.; Gell, M.; Wei, M. Apatite formation on alkaline-treated dense $\mathrm{TiO} 2$ coatings deposited using the solution precursor plasma spray process. Acta Biomater. 2008, 4 (3), 553-559.

(32) He, J.; Zhou, W.; Zhou, X.; Zhong, X.; Zhang, X.; Wan, P.; Zhu, B.; Chen, W. The anatase phase of nanotopography titania plays an important role on osteoblast cell morphology and proliferation. J. Mater. Sci.: Mater. Med. 2008, 19 (11), 3465-3472.

(33) Sjöström, T.; Dalby, M. J.; Hart, A.; Tare, R.; Oreffo, R. O.; Su, B. Fabrication of pillar-like titania nanostructures on titanium and their interactions with human skeletal stem cells. Acta Biomater. 2009, 5 (5), 1433-1441.

(34) Dalby, M.; Giannaras, D.; Riehle, M.; Gadegaard, N.; Affrossman, S.; Curtis, A. Rapid fibroblast adhesion to $27 \mathrm{~nm}$ high polymer demixed nano-topography. Biomaterials 2004, 25 (1), 7783.

(35) Junqueira, L. C. U.; Bignolas, G.; Brentani, R. R. Picrosirius staining plus polarization microscopy, a specific method for collagen detection in tissue sections. Histochem. J. 1979, 11 (4), 447-455.

(36) Yang, J.; McNamara, L. E.; Gadegaard, N.; Alakpa, E. V.; Burgess, K. V.; Meek, R. D.; Dalby, M. J. Nanotopographical induction of osteogenesis through adhesion, bone morphogenic protein cosignaling, and regulation of microRNAs. ACS Nano 2014, 8 (10), 9941-9953. 